\section{Testosteron-Gel macht Mann ehrlich}

Nach einem Verhaltensexperiment mit 91 Männern, kamen Bonner Neurologen zu dem Schluss, dass Testosteron ehrlicher macht. Die Tester spielten ein Würfelspiel bei dem es Geld zu gewinnen gab. Dabei durfte geschummelt werden. Im Anschluss wurde evaluiert, wie häufig getrickst wurde. Probanden, die vor dem Versuch mit Testosteron-Gel behandelt wurden, logen deutlich seltener als jene, die nur ein Placebo erhalten hatten. Das Hormon scheint den Stolz und das Bedürfnis, ein positives Selbstbild zu entwickeln, zu steigern.

Wibral M et al, PloS ONE 2012; doi: 10.1371/journal. pone.0046774

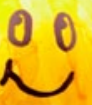

\section{Frühstücksei exkulpiert}

Ein Ei täglich hat weder

Einfluss auf das Schlaganfallnoch auf das Herzinfarktrisiko, bestätigt eine Metaanalyse, in die Daten von über 260.000 Patienten eingingen. Das relative Risiko, eine KHK zu erleiden, lag bei Konsum von einem Ei täglich bei 0,99, für einen Schlaganfall bei 0,91. Eine Subgruppenanalyse mit Diabetes-Patienten zeigte allerdings einen negativen Effekt aufs Herz: Die Teilnehmer mit dem höchsten Eierkonsum hatten ein um den Faktor 1,54 erhöhtes Risiko einer KHK. Die Autoren schränken jedoch ein, dass nur wenige Diabetiker-Studien ausgewertet werden konnten.

Rong Y et al, BMJ 2013, 346:e8539

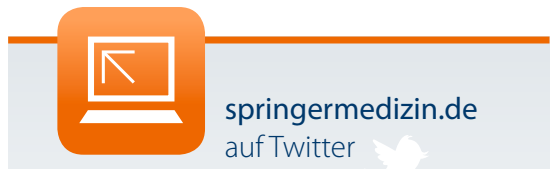

Auch in der fünften Jahreszeit heißt es:

"Lauschen" Sie unserem

Zwitschern und werden Sie zum

„Follower".

- twitter.com/springermedizin

Von wegen Schlummertrunk

\title{
Alkohol weckt auf!
}

Wer sich abends vom Alkohol beduselt in die Kissen kuschelt, schläft zwar rascher ein. Doch der Schlummer ist nicht von langer Dauer, wie die Ergebnisse einer Übersichtsstudie gezeigt haben.

Ein Team von englischen und kanadischen Schlafmedizinern hat sich in einer Übersichtsstudie mit dem Thema befasst, welche Wirkungen Alkoholkonsum vor dem Zubettgehen auf die Schlafqualität hat. Dabei stellte sich heraus: Beschwipst schläft man tatsächlich schneller ein. Doch in der zweiten Nachthälfte muss man diesen vermeintlichen Vorteil mit gehäuften Wachphasen büßen.

\section{Zweischneidige Wirkung}

Die Schlafzeit nimmt dadurch insgesamt ab. Tiefschlafphasen treten in der ersten Nachthälfte vermehrt auf. Die Wirkungen in der zweiten Hälfte des Schlafs sind dosisabhängig. Signifikant längerer Tiefschlaf findet sich bei hohen Dosen, wor- unter Trinkmengen ab etwa $60 \mathrm{~g}$ Alkohol zu verstehen sind. Das entspricht vier Standarddrinks, also rund 1,51 Bier bzw. $600 \mathrm{ml}$ Wein.

\section{REM-Suppression überraschte}

Eine Unterdrückung der REM-Schlafphasen konnten die Forscher für kleine Mengen Alkohol - bis zu zwei Standarddrinks - nicht nachweisen. Damit war nicht zu rechnen; bisher galt die REMSuppression als der Alkoholeffekt schlechthin, und zwar in allen Dosierungen. Mäßige oder hohe Dosen allerdings wirkten sich deutlich aus: Der Anteil der REM-Phasen am Gesamtschlaf sank, und die Zeit bis zur ersten REM-Phase verlängerte sich.

In die Studie waren Resultate von 20 Publikationen eingeflossen, an denen insgesamt 517 freiwillige Trinker teilgenommen hatten.

(rb)

Ebrahim 10 et al, Alcohol Clin Exp Res 2013 (online first)

\section{Überraschend häufig gutartig}

\section{App oder Arzt besser beim Melanom-Check?}

US-Dermatologen haben Smartphone-Apps zur Hautkrebs-Diagnostik getestet. In den meisten Fällen war die Trefferquote alles andere als zufriedenstellend.

Vier Smartphone-Apps, die alle vorgaben, bösartige Hautveränderungen zu erkennen, haben Hautärzte von der University of Pittsburgh geprüft. Zwei der Programme stuften $30 \%$, eines sogar mehr als $90 \%$ der Melanome als gutartig ein. Das vierte Programm erreichte in der Melanomdiagnostik eine Sensitivität von $98,1 \%$ - allerdings wurde die Diagnose dabei binnen 24 Stunden von einem Dermatologen gestellt, an den das Programm die Befundung des fotografierten Mals delegierte. Insgesamt wurden Fotos von 188 Hautläsionen mit den Smartphones beurteilt. Die App mit der höchsten Sensitivität der Melanomerkennung, war zugleich die teuerste: Pro beurteiltes Mal (!) wurde eine Gebühr von 5\$ fällig.

Wer sich auf eine App verlässt, statt zum Hautarzt zu gehen, sollte bedenken, dass viele der smarten Programme so smart gar nicht sind. Es gibt dafür auch keine behördliche Aufsicht, wie Wolf und Kollegen betonen. Schaden für die Betroffenen durch verzögerte Diagnosen lasse sich daher nicht ausschließen. (rb)

Wolf JA et al, JAMA Dermatology 2013 (online first) 